Neurosurg Focus 28 (2):E9, 2010

\title{
Musical hallucinations following insular glioma resection
}

\author{
Case report \\ Gustavo Rassier Isolan, M.D., Ph.D., ${ }^{1}$ Marino Muxfeldt Bianchin, M.D., Ph.D., 2 \\ José Augusto Bragatti, M.D., ${ }^{2}$ Carolina Torres, M.D., 2 \\ and Gilberto Schwartsmann, M.D., Ph.D. ${ }^{3}$
}

Departments of ${ }^{I}$ Neurosurgery, ${ }^{2}$ Neurology, and ${ }^{3}$ Oncology, Hospital de Clínicas de Porto Alegre, Universidade Federal do Rio Grande do Sul, Porto Alegre, Brazil

\begin{abstract}
Hallucinations can be auditory, visual, tactile, gustatory, or olfactory, and can be caused by psychiatric (such as schizophrenia and depression), neurological (such as cerebrovascular accidents, neoplasia, and infection), or endocrine and metabolic disorders. Musical hallucinations related to neurological disorders are rare. The authors present a case of a patient with a right insular glioma who developed transient musical hallucinations after microsurgical resection of the tumor. (DOI: 10.3171/2009.12.FOCUSFOCUSO9243)
\end{abstract}

KeY WordS • insula • musical hallucination • glioma

$\mathrm{H}$ ALLUCINATIONS are perception disorders characterized by the sensation of an external stimulus without the presence of such a stimulus. The term hallucination was introduced in the psychiatric literature in 1837 by Esquirol. ${ }^{23}$ Hallucinations can be auditory, visual, tactile, gustatory, or olfactory, and can be caused by psychiatric (such as schizophrenia and depression), neurological (such as cerebrovascular accidents, neoplasia, and infection), or endocrine and metabolic disturbances. ${ }^{1-4,6,11-15,17-20}$

Music and neuroscience have been related for a long time. In 1977, Critchley and Henson published the book Music and the Brain. Despite countless advances in this field during the last 30 years, Music and the Brain remains one of the main books on this topic. ${ }^{23}$ Auditory hallucinations in which music is perceived are rare and occur mainly in cases of deafness or psychiatric disorders. ${ }^{1}$ A small number of cases are related to neurological disorders, generally due to alterations of the primary auditory area. $3,4,17,18$

Insular gliomas are usually slow-growing tumors of low-grade malignancy. ${ }^{25-27}$ They may grow large before a diagnosis is made..$^{25-27}$ The main symptoms of these tumors are complex partial seizures with or without secondary generalization. ${ }^{25-27}$ Due to the deep localization and the close relationship with the MCA, tumor resection in insular topography is considered a highly complex surgical procedure.

Abbreviations used in this paper: $\mathrm{MCA}=$ middle cerebral artery.
In this paper we report the case of a patient who underwent microsurgical resection of a large right insular tumor and who developed musical hallucinations of limited duration during the early postoperative period. As far as we know, this is the first report of a patient with musical hallucinations after insular tumor resection.

\section{Case Report}

History and Presentation. This right-handed 34year-old man was first seen at our institution due to new onset of episodic intense headaches and 1 episode of complex partial seizures. The neurological examination was unremarkable. A CT scan and 1.5-T MR imaging with Gd (T1-weighted, T2-weighted, FLAIR, perfusion, and tractography) disclosed a nonenhanced intraaxial tumor in the right insula, extending to the superior temporal gyrus and enveloping the planum polare and planum temporale (Fig. 1).

Operation and Postoperative Course. The patient underwent resection of the tumor (>90\%) using microsurgical techniques, and underwent monitoring for somatosensory evoked potentials and transcranial electric motor evoked potentials. Pathological examination of the tumor specimen revealed a WHO Grade II glioma (Fig. 2). The surgical approach was conducted via a right frontotemporal craniotomy, using an interfacial dissection of the temporal fascia and microdissection of the sylvian fissure (transsylvian approach). ${ }^{16}$ The tumor was aspirated 

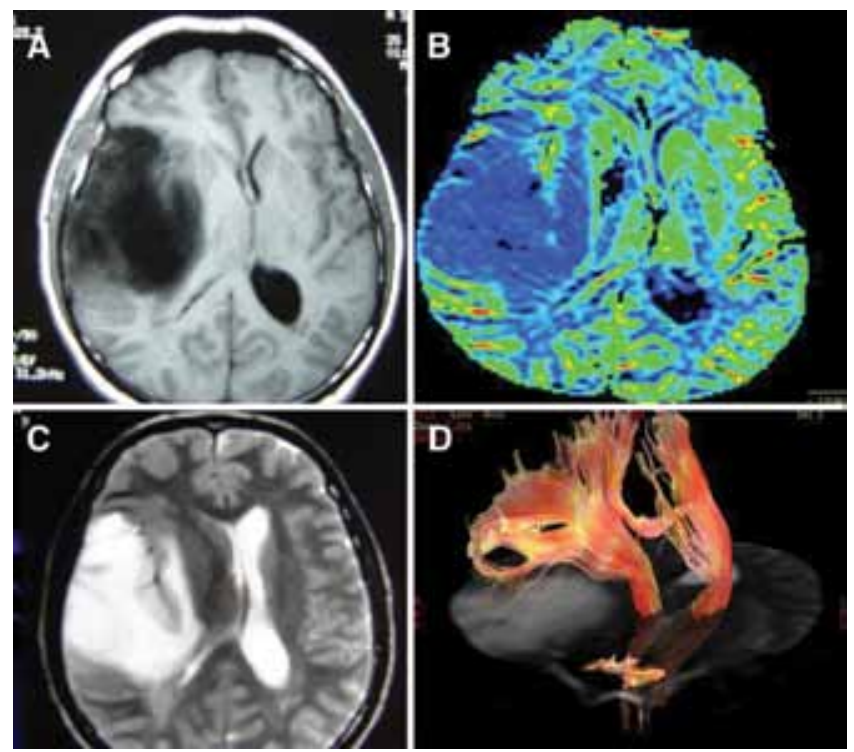

FIG. 1. Preoperative MR images of the patient. A: Contrast-enhanced T1-weighted image showing a nonenhanced lesion in the right insula causing midline deviation. B: Perfusion-weighted image showing the lesion. C: Contrast-enhanced T2-weighted image showing the absence of an important vasogenic edema. D: Diffusion imaging tractography showing the relation of the corticospinal tract to the tumor.

between the $\mathrm{M}_{2}$ and $\mathrm{M}_{3}$ segments of the MCA. The tumor portion extending to the planum polare and planum temporale was resected through the inferior circular sulcus of the insula, and a transopercular approach was used at the superior temporal gyrus, which was necessary to resect tumor in the lateral part of the Heschl gyrus. Deep resection of the tumor close to the internal capsule was stopped when there was an alteration in the intraoperative motor evoked potential.

The patient did not develop any neurological deficit as a result of the surgery. Diphenylhydantoin was initiated in the immediate preoperative period as prophylaxis for seizures. After 1 month, and while still receiving 100 $\mathrm{mg}$ of diphenylhydantoin three times a day, the patient began to have musical hallucinations. These hallucinations were characterized by a song that was very familiar to the patient although he was not able to identify it. The hallucinations occurred several times during the day, lasted several seconds, and were not associated with alterations of consciousness. This phenomenon occurred daily for approximately 2 months, and then disappeared. An electroencephalogram obtained at this time revealed discharges in the right centroparietal region and in the temporal regions of the right hemisphere. The patient has been asymptomatic for 1 year after surgery.

\section{Discussion}

Musical hallucinations can be observed to occur in association with otologic, psychiatric, and neurological causes, or their combination. Sporadic cases have been reported in patients with psychiatric, otorhinolaryngologic, or neurological problems..$^{1-4,6,11-15,17-20}$ It has been assumed that the upper portion of the temporal lobe is
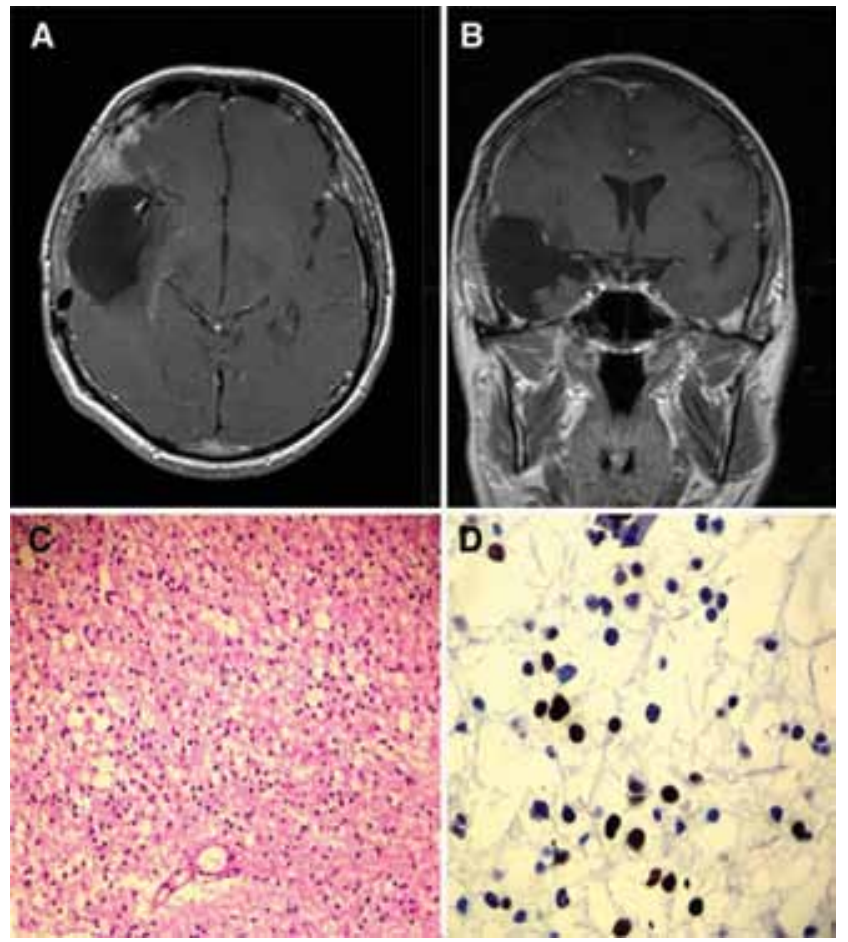

FIG. 2. Postoperative contrast-enhanced MR images ( $A$ and $B$ ) and photomicrographs (C and D). A: Axial T1-weighted image obtained 3 months after surgery showing tumor resection (> 90\%) with a small remaining tumor in the posterosuperior region of the tumor cavity. B: Coronal T1-weighted image showing the tumor resection. C: Tumor specimen showing features of a WHO Grade II astrocytic neoplasm. $\mathrm{H}$ $\&$ E. Original magnification $\times 100$. D: Immunohistochemical specimen showing $<2 \%$ staining for Ki 67 (MIB-1). Original magnification $\times 400$.

the etiopathogenic substrate of this phenomenon. The functional imaging studies using the paradigm "listen to music" result in a bilateral activation of the brain..$^{21,23} \mathrm{On}$ the other hand, studies of patients with acquired amusia suggest that the structures involved in this activity are the superior temporal cortex, the insula, and the frontal lobe of the nondominant cerebral hemisphere. ${ }^{23}$ Although less common, lesions in the dominant cerebral hemisphere can also cause deficits in musical perception. For the latter aspect, however, we should consider the bias that patients with lesions in the left hemisphere generally present with aphasia, which makes it more difficult to perform nonlinguistic ability tests. ${ }^{23}$

Musical hallucinations can involve 3 large groups of alterations: otologic, psychiatric, and less commonly, neurological alterations. Regarding this last group, our case is the first report of musical hallucinations involving resection of a tumor in the insula topography.

Musical hallucinations associated with deafness have an estimated prevalence of approximately $2.5 \%$ in older patients. ${ }^{6}$ Usually patients state that they hear familiar sounds, such as popular music. ${ }^{13,24}$ Sometimes a humming sound precedes the musical hallucination. Hearing musical instruments and voices is a common phenomenon. Based on the humming that developed into musical hallucinations, Gordon ${ }^{12}$ proposed that the etiological basis of the phenomenon is in the cochlea, although it is impos- 


\section{Musical hallucinations following insular glioma resection}

sible to confirm that the mechanism has been explained based only on this premise. The release hallucinations concept is based on West's perceptive theory, whose main hypothesis is that blindness and deafness interrupt external sensorial stimuli necessary to inhibit brain memory evocation. $., 7,22,23$ This phenomenon will release previously stored perceptions and visual or auditory memories. ${ }^{3,4}$ Release musical hallucinations occur mainly in the elderly with hearing deficiencies, mainly while awakening. The atypical antipsychotic drug quetiapine improved these symptoms in some patients. ${ }^{7}$ A similar phenomenon could be the reason for the transient symptoms experienced by our patient; the surgical procedure may have been responsible for the transitory release of the primary auditory area. Musical hallucinations associated with psychiatric disease are less common than verbal auditory hallucinations observed in schizophrenic, depressed, obsessive-compulsive, and alcoholic patients. Musical hallucinations are generally considered rare in psychiatric patients. ${ }^{3}$ Fukunishi et al. ${ }^{11}$ identified only 6 patients with musical hallucinations ( 3 of whom were deaf) in a population of 3578 psychiatric patients. However, this estimate may not be accurate, because Hermesh et al. ${ }^{14}$ identified $20 \%$ of 190 psychiatric patients (mainly patients with obsessive-compulsive problems) who developed musical hallucinations at some period in their life.

Musical hallucinations associated with neurological diseases are less frequent than in the 2 former groups. In case reports, they are usually associated with abnormalities of the right nondominant cerebral hemisphere. Although quite rare, there are reports of musical hallucinations associated with dementia, meningioma, ${ }^{19}$ gliomas, ${ }^{9}$ subarachnoid hemorrhage, ${ }^{20}$ cerebral ischemia, ${ }^{2}$ and pontine hemorrhage. ${ }^{18}$ To the best of our knowledge, there are no previous reports of hallucinations associated with insular gliomas.

On the other hand, the auditory aura is a rare phenomenon. In the series of 8000 patients of Florindo et al..$^{10}$ the auditory aura was present in only 121 cases. The role of laterality in epilepsy, where aura musical hallucinations occur, is a mechanism that must still be elucidated. The clinical characteristics of the case reported, in association with the electroencephalographic findings and the resection of the tumor tissue at the level of the planum temporale (more specifically, the anterior transverse temporal gyrus [Heschl gyrus]), lead us to believe the hypothesis that the postoperative musical hallucinations experienced by our patient could be a simple partial seizure due to surgical manipulation of the planum temporale or a release phenomenon.

Insular gliomas are tumors that become very large in many patients before they become symptomatic. ${ }^{25-27}$ Sometimes these tumors are a casual finding observed during MR imaging. The cortex of the insula is an interface between the allocortex and the neocortex and constitutes part of the paralimbic system. It is related to motor, sensorial, language, auditory-vestibular, and cognitive functions. ${ }^{8}$ The surgical treatment of these lesions is technically challenging because of their proximity to the internal capsule and the close anatomical relationship with the segments of the MCA and its branches. ${ }^{25-27}$ The
MR imaging tractography performed in this case illustrates the close proximity of the deep tumor portion to the corticospinal tract.

\section{Disclosure}

The authors report no conflict of interest concerning the materials or methods used in this study or the findings specified in this paper.

Author contributions to the study and manuscript preparation include the following. Conception and design: GR Isolan. Acquisition of data: GR Isolan, JA Bragatti, C Torres. Analysis and interpretation of data: GR Isolan, MM Bianchin. Drafting the article: GR Isolan, MM Bianchin. Critically revising the article: MM Bianchin, G Schwartsmann. Reviewed final version of the manuscript and approved it for submission: G Schwartsmann.

\section{References}

1. Ali JA: Musical hallucinations and deafness: a case report and review of the literature. Neuropsychiatry Neuropsychol Behav Neurol 15:66-70, 2002

2. Augustin J, Guegan-Massardier E, Levillain D, Lemarchand M, Mihout B, Hannequin D: [Musical hallucinosis following infarction of the right middle cerebral artery]. Rev Neurol (Paris) 157:289-292, 2001 (Fr)

3. Berrios GE: Musical hallucinations. A historical and clinical study. Br J Psychiatry 156:188-194, 1990

4. Berrios GE: Musical hallucinations: a statistical analysis of 46 cases. Psychopathology 24:356-360, 1991

5. Brown S, Martinez MJ, Parsons LM: Passive music listening spontaneously engages limbic and paralimbic systems. Neuroreport 15:2033-2037, 2004

6. Cole MG, Dowson L, Dendukuri N, Belzile E: The prevalence and phenomenology of auditory hallucinations among elderly subjects attending an audiology clinic. Int J Geriatr Psychiatry 17:444-452, 2002

7. David RR, Fernandez HH: Quetiapine for hypnogogic musical release hallucinations. J Geriatr Psychiatry Neurol 13:210211,2000

8. Duffau H, Capelle L, Lopes M, Faillot T, Sichez JP, Fohanno D: The insular lobe: physiopathological and surgical considerations. Neurosurgery 47:801-811, 2000

9. Evers S, Ellger T, Ringelstein EB, Knecht S: Is hemispheric language dominance relevant in musical hallucinations? Two case reports. Eur Arch Psychiatry Clin Neurosci 252:299_ 302,2002

10. Florindo I, Bisulli F, Pittau F, Naldi I, Striano P, Striano S, et al: Lateralizing value of the auditory aura in partial seizures. Epilepsia 47 (Suppl 5):68-72, 2006

11. Fukunishi I, Horikawa N, Onai H: Prevalence rate of musical hallucinations in a general hospital setting. Psychosomatics 39:175-178, 1998

12. Gordon AG: Do musical hallucinations always arise from the inner ear? Med Hypotheses 49:111-122, 1997

13. Griffiths TD: Musical hallucinosis in acquired deafness. Phenomenology and brain substrate. Brain 123:2065-2076, 2000

14. Hermesh H, Konas S, Shiloh R, Dar R, Marom S, Weizman A, et al: Musical hallucinations: prevalence in psychotic and nonpsychotic outpatients. J Clin Psychiatry 65:191-197, 2004

15. Keshavan MS, Kahn EM, Brar JS: Musical hallucinations following removal of a right frontal meningioma. J Neurol Neurosurg Psychiatry 51:1235-1236, 1988

16. Krayenbühl N, Isolan GR, Hafez A, Yaşargil MG: The relationship of the fronto-temporal branches of the facial nerve to the fascias of the temporal region: a literature review applied to practical anatomical dissection. Neurosurg Rev 30:8-15, 2007 
17. Mahendran R: The psychopathology of musical hallucinations. Singapore Med J 48:68-70, 2007

18. Murata S, Naritomi H, Sawada T: Musical auditory hallucinations caused by a brainstem lesion. Neurology 44:156-158, 1994

19. Nagaratnam N, Virk S, Brdarevic O: Musical hallucinations associated with recurrence of a right occipital meningioma. Br J Clin Pract 50:56-57, 1996

20. Paquier P, van Vugt P, Bal P, Cras P, Parizel PM, van Haesendonck J, et al: Transient musical hallucinosis of central origin: a review and clinical study. J Neurol Neurosurg Psychiatry 55:1069-1073, 1992

21. Sacks O: The power of music. Brain 129:2528-2532, 2006

22. Staley K, Hellier JL, Dudek FE: Do interictal spikes drive epileptogenesis? Neuroscientist 11:272-276, 2005

23. Stewart L, von Kriegstein K, Warren JD, Griffiths TD: Music and the brain: disorders of musical listening. Brain 129:25332553,2006
24. Warner N, Aziz V: Hymns and arias: musical hallucinations in older people in Wales. Int J Geriatr Psychiatry 20:658-660, 2005

25. Yaşargil MG: Microneurosurgery. Stuttgart: Thieme, Vol 4B, 1996, pp 262-276

26. Yaşargil MG, Cravens GF, Roth P: Surgical approaches to "inaccessible" brain tumors. Clin Neurosurg 34:42-110, 1988

27. Yaşargil MG, Krisht AF, Türe U, Al-Mefty O, Yaşargil DCH: Microsurgery of insular gliomas. Part I-IV. Contemp Neurosurg 24:(11):1-8; (12): 1-6; (13):1-6; (14):1-8, 2002

Manuscript submitted October 23, 2009.

Accepted December 7, 2009.

Address correspondence to: Gustavo Rassier Isolan, M.D., Ph.D., Department of Neurosurgery, Hospital de Clínicas de Porto Alegre, Universidade Federal do Rio Grande do Sul, Porto Alegre, Brazil. email: gisolan@yahoo.com.br. 